\title{
Link Types under Twisting Solid Tori with Essential Boundaries
}

\author{
TORU IKEDA
}

\begin{abstract}
Let $L$ be a link in $S^{3}$, and $V_{1}, \ldots, V_{l}$ solid tori with $\partial V_{i}$ being mutually disjoint non-paralell and essential in the exterior of $L$. We twist $V_{i}$, one by one to the meridional direction, and produce a new link $L^{\prime}$. The problem is the difference between two link types $L$ and $L^{\prime}$.
\end{abstract}

In the case of knots, some results were got in [12]. We will generalize them for the case of links.

\section{INTRODUCTION}

Throughout this paper, int $X$ and $N(X, Y)$ denotes the interior of $X$ and the neighbourhood of $X$ in $Y$ respectively.

Let $L_{1}$ and $L_{2}$ be unoriented links in the oriented sphere $S^{3}$. If there exists an orientation preserving homeomorphism of $S^{3}$ carrying $L_{1}$ to $L_{2}$, then we write $L_{1} \cong L_{2}$. This is equivalent to saying that $L_{1}$ and $L_{2}$ are ambient isotopic in $S^{3}$.

Let $L$ be a link in $S^{3}$, and $V$ a solid torus with a preferred framing such that $\partial V \cap L=\emptyset$. The wrapping number $\omega_{V}(L)$ of $L$ in $V$ is the 
minimal geometric intersection number of $L \cap V$ and a meridian disk of $V$. Suppose $\omega_{V}(L) \geq 2$ and $\partial V$ is oriented. Let $f^{(n)}: S^{3} \rightarrow S^{3}$ be a map, which is discontinuous on $\partial V$, such that $\left.f^{(n)}\right|_{S^{3}-V}$ is the identity, and $\left.f^{(n)}\right|_{V}$ is an orientation preserving self-homeomorphism of $V$ satisfying $f_{*}^{(n)}(m)=m$ and $f_{*}^{(n)}(l)=l+n m$, where $f_{*}^{(n)}: H_{1}(\partial V) \rightarrow H_{1}(\partial V)$ is an isomorphism induced by $f^{(n)}, m$ and $l$ are homology classes of a meridian and a preferred longitude of $\partial V$ with intersection number $m \cdot l=1$ respectively. Note that for a given link $L$, a solid torus $V$, an orientation of $\partial V$ and an integer $n$ determine a unique link type $f^{(n)}(L)$. We call $f^{(n)}$ an $n$-twist along $V$.

Let $V_{i}(i=1,2)$ be solid tori with mutually disjoint boundaries, $f_{i}^{(n)}$ be an $n$-twist along $V_{i}$. Since $f_{2}^{(n)}$ gives a homeomorphism on either $V_{1}$ or $S^{3}-i n t V_{1}, f_{2}^{(n)}\left(V_{1}\right)$ is a solid torus bounded by $f_{2}^{(n)}\left(\partial V_{1}\right)$ for any $n$. We do not distinguish notationally between $f_{1}^{\left(n_{1}\right)}$ and $n_{1}$-twist along $f_{2}^{\left(n_{2}\right)}\left(V_{1}\right)$. Similarly, denote $n_{2}$-twist along $f_{1}^{\left(n_{1}\right)}\left(V_{2}\right)$ by $f_{2}^{\left(n_{2}\right)}$. So we have always $f_{1}^{\left(n_{1}\right)} \circ f_{2}^{\left(n_{2}\right)}=f_{2}^{\left(n_{2}\right)} \circ f_{1}^{\left(n_{1}\right)}$ for any $n_{1}$ and $n_{2}$.
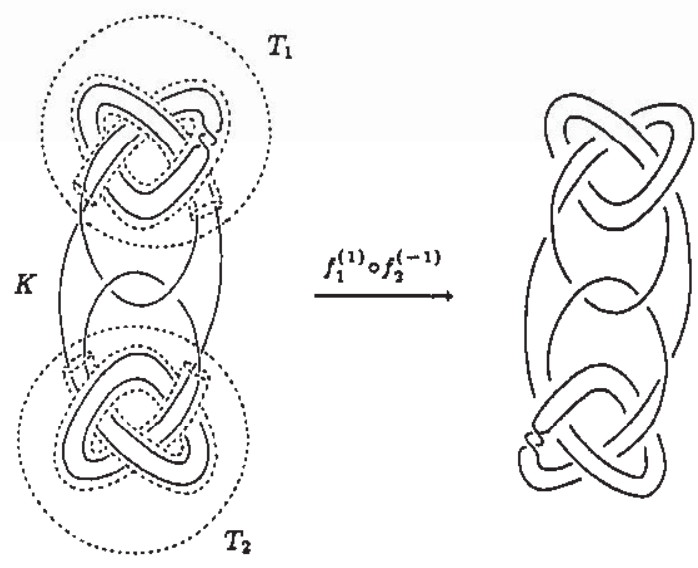

Figure 1. 
The link types do not always vary under some composition of twist maps. For example, the knot type $K$ in Figure 1 is invariant under $f_{1}^{(1)} \circ f_{2}^{(-1)}$. Then, when the link types varies?

In the case of knots, the following theorem is given by M. Kouno, K. Motegi and T. Shibuya ([KMS1], [KMS2]).

Theorem 1.1. Let $K$ be a knot in $S^{3}, V_{1}$ and $V_{2}$ solid tori with the boundaries being mutually disjoint and essential in the exterior of $K, f_{i}^{(n)}$ an $n$-twist along $V_{i}$. Suppose $K \subset V_{1} \subset V_{2}, \omega_{V_{1}}(K) \geq 2$ and $\omega_{V_{2}}\left(\right.$ coreV $\left._{1}\right) \geq 2$. If $\left(n_{1}, n_{2}\right) \neq(0,0)$, then $f_{1}^{\left(n_{1}\right)}(K) \not f_{2}^{\left(n_{2}\right)}(K)$.

Here core $V$ denotes a core of $V$. They also got some results about twisting of knots along a standard solid torus. Moreover, the author ([I2]) showed the following theorem.

Theorem 1.2. Let $K$ be a knot in $S^{3}, V_{1}, \ldots, V_{k}$ be solid tori with the boundaries being mutually disjoint, non-parallel and essential in the exterior of $K$, and $f_{i}^{(n)}$ an $n$-twist along $V_{i}$. If $\sum_{i=1}^{k} n_{1} \neq 0$, then $f_{1}^{\left(n_{1}\right)}$ 。 $\cdots \circ f_{k}^{\left(n_{k}\right)}(K) \not F K$.

As a special case of Theorem 1.2, he generalized Theorem 1.1 as follows ([I2]).

Theorem 1.3. Let $K$ be a knot in $S^{3}, V_{1}, \ldots, V_{k}$ be solid tori with the boundaries being mutually disjoint, non-parallel and essential in the exterior of $K$, and $f_{i}^{(n)}$ an n-twist along $V_{i}$. Suppose $K \subset V_{1} \subset \cdots \subset$ $V_{k}, \omega_{V_{1}}(K) \geq 2$, and $\omega_{V_{i}}\left(\operatorname{coreV}_{i-1}\right) \geq 2$ for $2 \leq i \leq k$. If $\left(n_{1}, \ldots, n_{k}\right) \neq$ $(0, \ldots, 0)$, then $f_{1}^{\left(n_{1}\right)} \circ \ldots \circ f_{k}^{\left(n_{k}\right)}(K) \not K$.

The problem in this paper is whether the translation of these results for the case of links are true or not.

\section{MAIN RESULTS}

Let $L$ be a non-separable non-trivial link in $S^{3}$. Consider the torus decomposition of $E=S^{3}-\operatorname{int} N\left(L, S^{3}\right)$ (see [JS] and [Jo]). By a finite set $\mathcal{T}_{D}^{\prime}$ of mutually disjoint, non-parallel, essential tori imbedded in $E$,we can decompose $E$ uniquely into the pieces each of which is Seifert 
fibered or admits a complete hyperbolic structure of finite volume in its interior (see [T]). We call the piece a Seifert piece or a hyperbolic piece respectively. Moreover each Seifert piece is one of a torus knot space, a cable space and a composing space (see [I1]).

Suppose $T_{1}, \ldots, T_{l} \in \mathcal{T}_{D}^{\prime}$ are tori, allowing duplication, each of which satisfies the following:

(1) There is a solid torus $V_{i}$ bounded by $T_{i}$ satisfying $\omega_{V_{i}}(L) \geq 2$.

(2) For any $T \in \mathcal{T}_{D}^{\prime}$ satisfying (1), $T_{i}=T$ for some $i$.

(3) $i \neq j$ implies $V_{i} \neq V_{j}$.

(4) If $T_{i}$ is standard and $\omega_{S^{3}-i n t V_{i}}(L) \geq 2$, then there is an integer $j \neq i$ such that $T_{i}=T_{j}$, i.e. $V_{i} \cap V_{j}=T_{i}$ and $V_{i} \cup V_{j}=S^{3}$.

Let $\mathcal{T}_{D}=\left\{T_{1}, \ldots, T_{i}\right\}$. Note that, by the property (4), we do not always have a one to one correspondence between indices $1, \ldots, l$ and the elements of $\mathcal{T}_{D}$. Suppose $T_{i}$ has an orientation decided by that of $S^{3}$ and a normal of $T_{i}$ oriented to the exterior of $V_{i}$. If there is an orientation preserving homeomorphism of $S^{3}$ carrying $V_{i}$ to $V_{j}$, it gives an orientation preserving homeomorphism from $T_{i}$ to $T_{j}$.

Let $f_{i}^{(n)}$ be an $n$-twist along $V_{i}$. First we show the following theorem.

Theorem 2.1. If $\sum_{i=1}^{l} n_{i} \neq 0$, then $f_{1}^{\left(n_{1}\right)} \circ \cdots \circ f_{l}^{\left(n_{l}\right)}(L) \neq L$.

Though Theorem 2.1 gives the translation of Theorem 1.3 for the case of links, it is not enough to see the general cases. First we remark the following case, which can never be found for any knots.

Remark 2.1. When $T_{i}$ bounds two different Seifert pieces $M$ and $M^{\prime}$ in both sides, the Seifert fibering of $M$ and $M^{\prime}$ cannot be extended over $T_{i}$. But fibers on $T_{i}=f_{i}^{(n)}\left(T_{i}\right)$ given by $f_{i}^{(n)}(M)$ and $f_{i}^{(n)}\left(M^{\prime}\right)$ can be mutually isotopic for some $n$. In this case, the Seifert fibering of $f_{i}^{(n)}(M)$ and $f_{i}^{(n)}\left(M^{\prime}\right)$ are extended over $T_{i}$. Thus $T_{i}$ is contained in the interior of a new Seifert piece $f_{i}^{\left(n_{i}\right)}(M) \cup f_{i}^{\left(n_{i}\right)}\left(M^{\prime}\right)$.

We show an example in Figure 2. Let $L$ be a link constructed from a Hopf link by $(5,2)$-cabling for one component and $(2,3)$-cabling for 
another. The torus decomposition of the exterior of $L$ gives an essential torus $T$. Let $V$ be a solid torus bounded by $T$ containing $(5,2)$-cable. The exterior $E$ of $L$ consists of two Seifert pieces. The orbit manifold of each piece is a disk with a hole and a singular point. Make a new link $L^{\prime}=f^{(-1)}(L)$ where $f^{(n)}$ is an $n$-twist along $V$. Since $L^{\prime}$ is a torus link of type $(6,4), T=f^{(-1)}(T)$ is contained in a Seifert piece with the orbit manifold being a disk with a hole and two singular points.

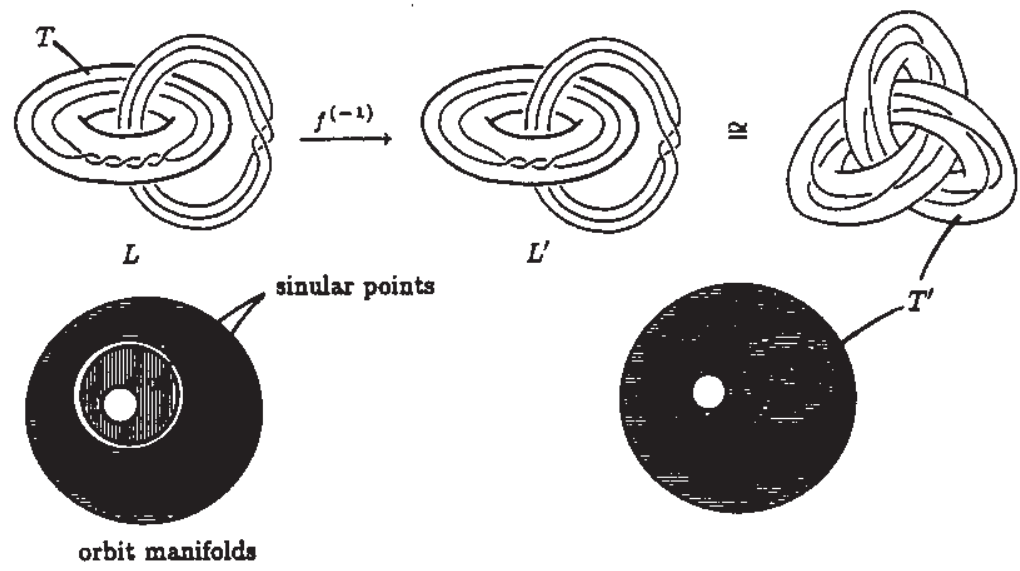

Figure 2.

In this case, there can be an essential torus $T^{\prime}$ which intersects $T$ for all deformation by isotopies of $f^{(-1)}(E)$. Let $V^{\prime}$ be a solid torus bounded by $T^{\prime}$ and $g^{(n)}$ an $n$-twist along $V^{\prime}$. We can define $g^{(n)} \circ f^{(-1)}$ for any $n \neq 0$, but this map is not considered in Theorem 2.1.

The non-parallel essential tori, which intersect each other for all deformation by isotopies in $E$, exist only in Seifert pieces. Let $\mathcal{T}_{S}^{\prime}$ be the set of mutually non-parallel essential tori, which are the representatives of all the isotopy classes of essential tori in the interior of the Seifert pieces. Suppose $T_{i+1}, \ldots, T_{m}$ are tori in $\mathcal{T}_{S}^{\prime}$, allowing duplication, which satisfy the following: 
(1) There is a solid torus $V_{i}$ bounded by $T_{i} \in \mathcal{T}_{S}$ satisfying $\omega_{V_{i}}(L) \geq$ 2.

(2) For any $T \in \mathcal{T}_{S}^{\prime}$ satisfying (1), $T_{i}=T$ for some $l+1 \leq i \leq m$.

(3) $i \neq j$ implies $V_{i} \neq V_{j}$.

(4) If $T_{i} \in T_{S}^{\prime}$ is standard and $\omega_{S^{3}-i n t V_{i}}(L) \geq 2$, then there is a torus $T_{j} \in T_{S}^{\prime}$ with $j \neq i$ and $T_{i}=T_{j}$, i.e. $V_{i} \cap V_{j}=T_{i}$ and $V_{i} \cup V_{j}=S^{3}$.

Let $\mathcal{T}_{S}=\left\{T_{l+1}, \ldots, T_{m}\right\}$. Tori $T_{i}$ and $T_{j}$ in $T_{S}$ are not always mutually disjoint, even if $T_{i} \neq T_{j}$. Let $\mathcal{T}=\mathcal{T}_{D} \cup \mathcal{T}_{S}$.

Our result in the general case is stated as follows. Any cases reduce to this theorem, which we show in $§ 5$.

Theorem 2.2. Let $\left\{T_{a(i)}\right\}_{i=1}^{k_{1}} \subset \mathcal{T}$ and $\left\{T_{b(j)}\right\}_{j=1}^{k_{2}} \subset \mathcal{T}$ are two sets of mutually disjoint tori. If $\sum_{i=1}^{k_{1}} n_{i}-\sum_{j=1}^{k_{2}} m_{j} \neq 0$, then $f_{a(1)}^{\left(n_{1}\right)} \circ \cdots \circ$ $f_{a\left(k_{1}\right)}^{\left(n_{k_{1}}\right)}(L) \not f_{b(1)}^{\left(m_{1}\right)} \circ \cdots \circ f_{b\left(k_{2}\right)}^{\left(m_{k_{2}}\right)}(L)$.

Hеге $T_{a(i)}$ may intersect $T_{b(j)}$ for some $i$ and $j$.

In a special case when all the solid tori to twist are in an inclusion relation, we get the following theorem.

Theorem 2.3. Let $L$ be a link in $S^{3}, V_{c(i)}(1 \leq i \leq k)$ solid tori such that $V_{c(1)} \subset V_{c(2)} \subset \cdots \subset V_{c(k)}, \omega_{V_{c(1)}}(L) \geq 2$ and $\omega_{V_{c(i+1)}}((L-$ $\left.\left.V_{c(i)}\right) \cup \operatorname{core} V_{c(i)}\right) \geq 2$ for $1 \leq i \leq k-1$. Then $f_{c(1)}^{\left(n_{1}\right)} \circ \cdots \circ f_{c(k)}^{\left(n_{k}\right)}(L) \neq L$ for any $\left(n_{1}, \ldots, n_{k}\right) \neq(0, \ldots, 0)$.

Theorem 2.3 gives the translation of Theorem 1.3.

\section{PIECES IN A SOLID TORUS}

Let $V$ be a torus in $S^{3}$ such that $T=\partial V$ is essential in $E, m$ and $l$ homology classes of a meridian and a preferred longitude of $T$ respectively. In the statement and the proof of Lemma 3.1, double signs are in the same order.

Lemma 3.1. Let $T$ be a fibered torus of type $(p, q)$ where $p$ and $q$ are coprime integers with $q \neq 0, F: T \rightarrow T$ a homeomorphism which 
preserves the orientation and the fibers, and $F_{*}: H_{1}(T) \rightarrow H_{1}(T)$ an isomorphism induced by $F$. If $F_{*}(m)= \pm m$, then $F_{*}(l)= \pm l$.

Proof. Since $F$ preserves the orientation of $T$ and $F_{*}$ is an isomorphism, we have $F_{*}(l)= \pm(l+\alpha m)$ for some $\alpha$. Moreover, since $F$ preserves the fibers on $T$, we have $F_{*}(p m+q l)= \pm(p m+q(l+\alpha m))=$ $\pm(p m+q l)$. Then $\alpha=0$, hence $F_{*}(l)= \pm l$.

Let $M$ be a piece got by the torus decomposition of $V-\operatorname{int} N(L \cap$ $V, V)$ attaching $T$ in $V$. In the statement and the proof of Lemma 3.2 , double signs are in the same order.

Lemma 3.2. Let $F: M \rightarrow M$ be an orientation preserving homeomorphism carrying $T$ to $T, F_{*}: H_{1}(T) \rightarrow H_{1}(T)$ an isomorphism induced by $F$. Suppose $F_{*}(m)= \pm m$. If $M$ is not a Seifert piece with meridional fibers on $T$, then $F_{*}(l)= \pm l$.

Proof. First, assume $M$ is a hyperbolic piece. We have $F_{*}(l)=$ $\pm(l+\alpha m)$ for some $\alpha$. By Mostow's rigidity theorem ([T, 5.7.4.]), $\operatorname{Isom}($ int $M) \cong O u t\left(\pi_{1}(\right.$ int $\left.M)\right)$ is a finite group. So $\left.F\right|_{\text {int } M}$ is homotopic to a unique isometry $G$ of $M$ and $G^{N}$ is the identity for some integer $N>0$. Then $F^{N}$ is homotopic to the identity. Then $F_{*}^{N}(l)=$ $( \pm l)^{N}(l+N \alpha m)=l$. Therefore $\alpha=0$, hence $F_{*}(l)= \pm l$.

Next, assume $M$ is a Seifert piece without meridional fibers on $T$. Since $T$ is an essential torus in $E, M$ is neither a trivial knot space nor a Hopf link space. By [11, Theorem 1], $M$ is homeomorphic to an exterior of a Seifert link. So $M$ is either a torus link space, a cable space or a composing space by [I1, Theorem 2]. Therefore $M$ has a unique Seifert fibering up to isotopy by [Ja, Lemma VI.17]. Then $F$ is isotopic to a fiber preserving homeomorphism. Hence the proof completes by Lemma 3.1 .

Assume $M$ is a Seifert piece with meridional fibers on $T$. Then $M$ is a composing space, because a Seifert piece in a link exterior is either a torus link space, a cable space or a composing space. In this case, there is a solid torus $V^{\prime}$ satisfying $V^{\prime} \cap M=T^{\prime}$ such that $T^{\prime}=\partial V^{\prime}$ has meridional fibers given by $M$ [I1, Proposition 12].

Lemma 3.3. Let $T^{\prime}, V^{\prime}$ be as above, $T^{\prime \prime}$ an essential torus in int $M, V^{\prime \prime}$ a solid torus bounded by $T^{\prime \prime}$ containing $V^{\prime}$, and $f^{(n)}, f^{\prime(n)}$, 
$f^{\prime \prime}(n) n$-twists along $V, V^{\prime}, V^{\prime \prime}$ respectively. Then we have $f^{(n)}(L) \cong$ $f^{\prime(n)}(L) \cong f^{\prime \prime(n)}(L)$.

Proof. By [11, Proposition 13], there is a companion of $L$, which is a composite link, with coreV' being one of its components. Since $\omega_{V}\left(\operatorname{core}^{\prime}\right)=1, f^{(n)}$ twists $V^{\prime} n$ times to the meridional direction. Moreover, since $\omega_{V}\left(L-V^{\prime}\right)=0, f^{(n)}\left(\left(L-V^{\prime}\right) \cup\right.$ coreV $\left.^{\prime}\right)$ is ambient isotopic to $\left(L-V^{\prime}\right) \cup$ core $V^{\prime}$ in $V$. So we get $f^{(n)}(L) \cong f^{\prime(n)}(L)$. Other cases are similar.

We show an example in Figure 3. By Lemma $3.3, f^{(n)}, f^{\prime(n)}$ and $f^{\prime \prime(n)}$ are mutually replaceable.

\section{PROOF OF THEOREM 2.1}

Let $m_{i}$ and $l_{i}$ be the homology classes of a meridian and a preferred longitude of $T_{i}$ with $m_{i} \cdot l_{i}=1$ respectively, $M_{i}$ the piece got by decomposing $E \cap V_{i}$ by $\mathcal{T}_{D}^{\prime}$ attaching $T_{i}$ in $V_{i}$.
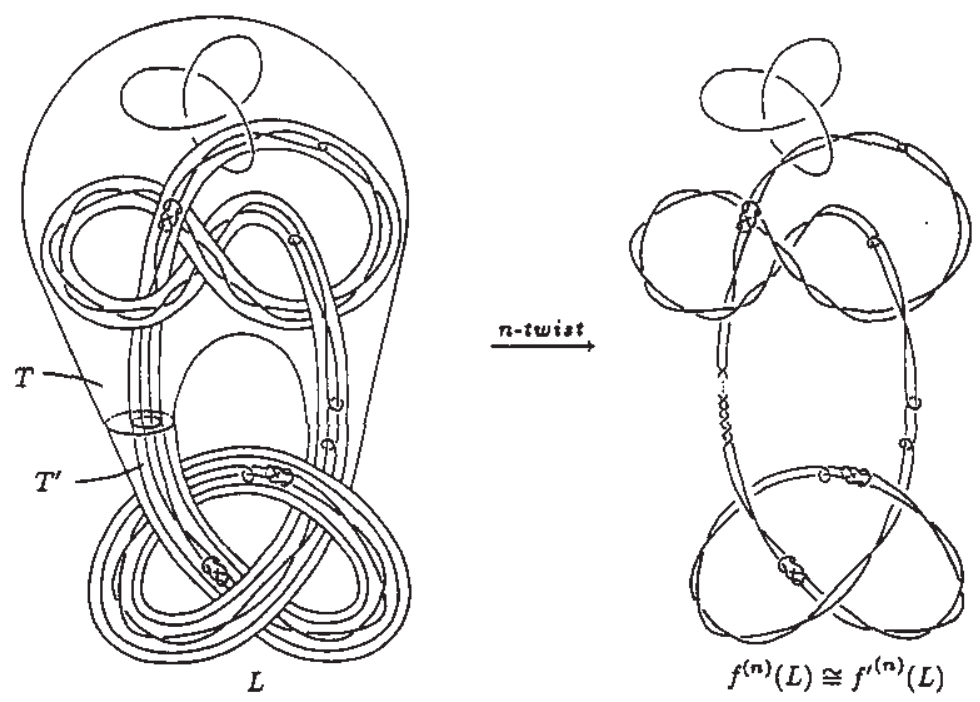

Figure 3. 
In this section, we suppose $T_{i} \in \mathcal{T}_{D}$.

Proof of Theorem 2.1. Assume $f_{1}^{\left(n_{1}\right)} \circ \cdots \circ f_{l}^{\left(n_{l}\right)}(L) \cong L$ for some $\left(n_{1}, \ldots, n_{l}\right)$. Under this assumption, we show $\sum_{i=1}^{l} n_{i}=0$ in the following.

There is an orientation preserving homeomorphism $\varphi: S^{3} \rightarrow S^{3}$ carrying $f_{1}^{\left(n_{1}\right)} \circ \cdots \circ f_{l}^{\left(n_{i}\right)}(L)$ to $L$. Assume some $M_{i}$ with $n_{i} \neq 0$ is a composing space with meridional fibers on $T_{i}$. Let $T_{j} \subset \partial M_{i}$ be a torus with meridional fibers such that $V_{j} \cap M_{i}=T_{j}$. By the condition of the torus decomposition of $E, M_{j}$ is not a composing space with meridional fibers on $T_{j}$. Change $f_{i}^{\left(n_{i}\right)}$ for $f_{j}^{\left(n_{i}\right)}$ by Lemma 3.3. Then we can assume $M_{i}$ with $n_{i} \neq 0$ is not a composing space with meridional fibers on $T_{i}$ for any $i$.

For convenience, denote $\varphi \circ f_{1}^{\left(n_{1}\right)} \circ \cdots \circ f_{l}^{\left(n_{l}\right)}$ by $\varphi_{\mu}$ where $\mu=$ $\left(n_{1}, \ldots, n_{l}\right)$. Although $\varphi_{\mu}$ is a discontinuous map on $S^{3}$, it gives an orientation preserving homeomorphism on each $M_{i}$, which keeps the hyperbolic structure or Seifert fibering. Thus $\left\{\varphi_{\mu}(T)\right\}_{T \in \mathcal{T}_{D}^{\prime}}$ is a set of essential tori in $E=\varphi_{\mu}(E)$, a subset of which gives a torus decomposition of $E$. As in Remark 2.1, $\varphi_{\mu}(T)$ can be contained in the interior of a Seifert piece for some $T \in \mathcal{T}_{D}^{\prime}$. In this case, the number of tori given by the torus decomposition decreases. This contradicts the uniqueness of the torus decomposition of $E$. Hence each torus $\varphi_{\mu}(T)$ is isotopic to a torus in $\mathcal{T}_{D}^{\prime}$. Then $\varphi_{\mu}\left(\bigcup \mathcal{T}_{D}\right)$ is isotopic to $\bigcup \mathcal{T}_{D}$. Modify $\varphi$ so as to satisfy $\varphi_{\mu}\left(\bigcup T_{D}\right)=\bigcup T_{D}$.

$\varphi_{\mu}$ induces a permutation $\emptyset$ on a finite set $\mathcal{T}_{D}$. We can write $\emptyset$ as a product disjoint cycles as follows:

$$
\left(T_{p(1,1)}, T_{p(1,2)}, \ldots, T_{p\left(1, N_{1}\right)}\right)\left(T_{p(2,1)}, \ldots, T_{p\left(2, N_{2}\right)}\right) \cdots\left(T_{p(r, 1)}, \ldots, T_{p\left(r, N_{r}\right)}\right)
$$

We call the set $\left\{T_{p(i, 1)}, \ldots, T_{p\left(i, N_{i}\right)}\right\}$ an orbit under $\varphi_{\mu}$.

$$
\text { Let } \nu_{i}=\sum_{j=1}^{N_{i}} n_{p(i, j)} \text {. Since } \sum_{i=1}^{l} n_{i}=\sum_{i=1}^{r} \nu_{i} \text {, following Lemma 4.1 }
$$


Lemma 4.1. Suppose $f_{1}^{\left(n_{1}\right)} \circ \cdots \circ f_{l}^{\left(n_{l}\right)}(L) \cong L$ and any $M_{i}$ with $n_{i} \neq 0$ is not a composing space with meridional fibers on $T_{i}$. Let $\varphi_{\mu}$ and the orbits under $\varphi_{\mu}$ are as above. Then we have $\nu_{i}=0$ for any $i$.

Proof. Let $N=\operatorname{LCM}\left\{N_{1}, \ldots, N_{r}\right\}$ and $\nu_{i}^{\prime}=\nu_{i} N / N_{i}$. We calculate $\varphi_{\mu}^{N}$ in the following. In the case $r=1$ and $N=N_{1}=l$, we have

$$
\begin{aligned}
\varphi_{\mu}^{l} & =\varphi_{\mu} \circ \varphi_{\mu}^{l-1} \\
& =\varphi \circ f_{p(1,1)}^{\left(n_{p(1,1)}\right)} \circ f_{p(1,2)}^{\left(n_{p(1,2)}\right)} \circ \cdots \circ f_{p(1, l)}^{\left(n_{p(1, l)}\right)} \circ \varphi_{\mu}^{l-1} .
\end{aligned}
$$

Using

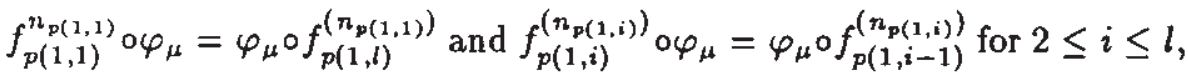

we get

$$
\begin{aligned}
& \varphi_{\mu}^{l}=\varphi \circ \varphi_{\mu} \circ f_{p(1, l)}^{\left(n_{p(1,1)}\right)} \circ f_{p(1,1)}^{\left(n_{p(1,2)}\right)} \circ \cdots \circ f_{p(1, l-1)}^{\left(n_{p(1, l)}\right)} \circ \varphi_{\mu}^{l-2} \\
& =\varphi^{2} \circ f_{p(1,1)}^{\left(n_{p(1,1)}+n_{p(1,2)}\right)} \circ f_{p(1,2)}^{\left(n_{p(1,2)}+n_{p(1,3)}\right)} \circ \cdots \circ f_{p(1,1)}^{\left.n_{p(1, l)}+n_{p(1,1)}\right)} \circ \varphi_{\mu}^{l-2} \\
& =\varphi^{3} \circ f_{p(1,1)}^{\left(n_{p(1,1)}+n_{p(1,2)}+n_{p(1,3)}\right)} \circ f_{p(1,2)}^{\left(n_{p(1,2)}+n_{p(1,3)}+n_{p(1,1)}\right)} \\
& \circ \cdots \circ f_{p(1, l)}^{\left(n_{p(1, l)}+n_{p(1,1)}+n_{p(1,2)}\right)} \circ \varphi_{\mu}^{l-3} \\
& =\varphi^{l} \circ f_{p(1,1)}^{\left(\nu_{1}\right)} \circ f_{p(1,2)}^{\left(\nu_{1}\right)} \circ \cdots \circ f_{p(1, l)}^{\left(\nu_{1}\right)} \text {. }
\end{aligned}
$$

Similarly, in the general case, we have

$$
\varphi_{\mu}^{N}=\varphi^{N} \circ\left(f_{p(1,1)}^{\left(\nu_{1}^{\prime}\right)} \circ \cdots \circ f_{p\left(1, N_{1}\right)}^{\left(\nu_{1}^{\prime}\right)}\right) \circ \cdots \circ\left(f_{p(r, 1)}^{\left(\nu_{r}^{\prime}\right)} \circ \cdots \circ f_{p\left(r, N_{r}\right)}^{\left(\nu_{r}^{\prime}\right)}\right) .
$$

The pieces $M_{p(i, j)}\left(1 \leq j \leq N_{i}\right)$ are mutually homeomorphic. So if $M_{p(i, 1)}$ is a composing space with meridional fibers on $T_{p(i, 1)}$, then $n_{p(i, j)}=0$ for $1 \leq j \leq N_{i}$, thus $\nu_{i}^{\prime}=\nu_{i}=0$. 
Assume that $M_{p(i, 1)}$ is not a composing space with meridional fibers on $T_{p(i, 1)} \cdot \varphi_{\mu}^{N}$ gives an orientation preserving self-homeomorphism of $M_{p(i, 1)}$ carrying $T_{p(i, 1)}$ to $T_{p(i, 1)}$. Note that when $V_{p(i, 1)} \subset$ int $V_{j}$ for some $j, V_{p(i, 1)}$ can be automatically twisted by $f_{j}^{\left(n_{j}\right)}$. So $\varphi_{\mu}^{N}$ induces an isomorphism of $H_{1}\left(T_{p(i, 1)}\right)$ carrying $m_{p(i, 1)}$ to $\pm m_{p(i, 1)}$ and $l_{p(i, 1)}$ to $\pm\left(l_{p(i, 1)}+\left(\nu_{i}^{\prime}+\alpha_{i}\right) m_{p(i, 1)}\right)$. Here $\alpha_{i}$ depends on the twists along the solid tori containing $V_{p(i, 1)}$. Applying Lemma 3.2 to $\left.\varphi_{\mu}^{N}\right|_{M_{p(i, 1)}}$, we get $\nu_{i}^{\prime}+\alpha_{i}=0$. If either there is no solid torus containing $V_{p(i, 1)}$ or any of the twists along the solid tori containing $V_{p(i, 1)}$ is 0 -twist, then $\alpha_{i}=0$. So we get $\alpha_{i}=\nu_{i}^{\prime}=0$ in order of the inclusion relation of the solid tori. Therefore we get $\nu_{i}=0$ for any $i$. This completes the proof of Lemma 4.1 and Theorem 2.1.

\section{PROOF OF THEOREM 2.2}

In this section, we suppose that $T_{i}$ does not always belong to $\mathcal{T}_{D}$.

Suppose $M_{i}$ and $M_{j}$ are Seifert pieces such that $M_{i} \cap M_{j}=T_{i}$. If a regular fiber on $T_{i}$ given by $M_{i}$ (resp. $M_{j}$ ) is homologous to ( $p+$ $n q) m_{i}+q l_{i}$ (resp. $p m_{i}+q l_{i}$ ) for some coprime integers $(p, q)$ and an integer $n$, then we say $T_{i}$ is a connectable torus and the discrepancy of $T_{i}$ is $n$. This means that the Seifert fibering of $M_{i}$ and $M_{j}$ are connectable after $(-n)$-twist along $V_{i}$.

Moreover, suppose $T_{i}$ is standard in $S^{3}$ and there is an integer $j$ such that $V_{i} \cap V_{j}=T_{i}=T_{j}$. If a regular fiber on $T_{i}$ given by $M_{i}$ (resp. $M_{j}$ ) is homologous to $\left(p+n_{1} q\right) m_{i}+q l_{i}$ (resp. $p m_{i}+\left(-n_{2} p+q\right) l_{i}$ ), then we say $T_{i}=T_{j}$ is a connectable torus and the discrepancies of $T_{i}$ and $T_{j}$ are $n_{1}$ and $n_{2}$ respectively. This means that the Seifert fibering of 
$M_{i}$ and $M_{j}$ are connectable after $\left(-n_{i}\right)$-twist along $V_{i}$ and $\left(-n_{j}\right)$-twist along $V_{j}$.

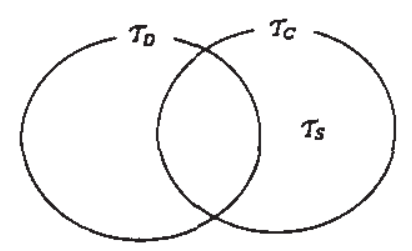

Figure 4.

Obviously, the discrepancy of a connectable torus is unique. Let $\mathcal{T}_{C}$ be the set of all the connectable tori in $\mathcal{T}$ (Figure 4.). Then we have $\mathcal{T}=\mathcal{T}_{D} \cup \mathcal{T}_{C}$ and $\mathcal{T}_{S}=\mathcal{T}_{C}-\mathcal{T}_{D}$.

Let $\left\{M_{\nu_{i}}\right\}_{i=1}^{r}$ be a set of Seifert pieces in $E$ which satisfies the following:

(1) $M=\bigcup_{i=1}^{r} M_{\nu_{i}}$ is connected.

(2) $T_{\nu_{i}} \subset$ int $M$ implies $T_{\nu_{i}} \in \mathcal{T}_{C}$ for any $i$.

(3) Fibers on $T_{\nu_{i}}$ given by $M_{\nu_{i}}$ are not meridional for any $i$.

Let $\mu_{i}$ be the discrepancy of $T_{\nu_{i}}$. By $\Phi=f_{\nu_{1}}^{\left(-\mu_{1}\right)} \circ \ldots \circ f_{\nu_{r}}^{\left(-\mu_{r}\right)}, \Phi(M)$ gets to be a connected Seifert manifold. In other words, $M$ is got by applying $\Phi^{-1}$ to the Seifert manifold $\Phi(M)$. So we call $M$ a twisted Seifert manifold. In this paper, we suppose the boundary components of the twisted Seifert manifolds always belong to $T \cup\{\partial E\}$. When 
a twisted Seifert manifold which contains $M$ is only $M$, we call $M$ a maximal twisted Seifert manifold. In Figure 2, the exterior of $L$ is an example of a maximal twisted Seifert manifold.

Lemma 5.1. Let $V$ be a solid torus with $T=\partial V$ being essential in $E$. Suppose there is a twisted Seifert manifold $M$ attaching $T$ in $V, F: M \rightarrow M$ is a fiber preserving map carrying $T$ to $T$ which may be discontinuous on some essential tori in int $M, F_{*}: H_{1}(T) \rightarrow H_{1}(T)$ is an isomorphism induced by $F, m$ and $l$ are homology classes of $a$ incridian and a preferred longitude of $T$ respectively. If $F_{*}(m)= \pm m$, then $F_{*}(l)= \pm l$ (The double signs are in the same order).

Proof. Since $T$ is essential in $E, M$ is neither a trivial knot space nor a Hopf link space, and so are the Seifert pieces in $M$. Then the Seifert fibering of each Seifert piece is unique by [Ja, Lemma VI.17]. Therefore the Seifert fibering of $T$ given by $M$ is unique and not meridional. Hence the proof completes by Lemma 3.1.

Let $\mathcal{T}_{\mu}^{\prime}$ be a set of mutually non-parallel essential tori, which are the representatives of all the isotopy classes in the exterior $E^{\prime}$ of $f_{1}^{\left(n_{1}\right)} 0 \ldots 0$ $f_{m}^{\left(n_{m}\right)}(L)$, where $\mu=\left(n_{1}, \ldots, n_{m}\right)$. Suppose any two tori in $\mathcal{T}_{\mu}^{\prime}$ have the transverse intersection with minimal number of components. Let $T_{1}^{\prime}, \ldots, T_{\nu}^{\prime}$ be tori in $\mathcal{T}_{\mu}^{\prime}$, allowing duplication, which satisfy the following:

(1) $T_{i}^{\prime}$ is not isotopic to $f_{1}^{\left(n_{1}\right)} \circ \ldots \circ f_{m}^{\left(n_{m}\right)}\left(T_{j}\right)$ for any $1 \leq i \leq \nu$ and $1 \leq j \leq m$.

(2) There is a solid torus $V_{i}^{\prime}$ bounded by $T_{i}^{\prime}$ satisfying $\omega_{V_{i}^{\prime}}(L) \geq 2$ for any $i$.

(3) For any $T \in \mathcal{T}_{\mu}^{\prime}$ satisfying (1) and (2), $T_{i}^{\prime}=T$ for some $i$.

(4) $i \neq j$ implies $V_{i}^{\prime} \neq V_{j}^{\prime}$.

(5) If $T_{i}^{\prime}$ is standard and $\omega_{S^{3}-i n t V_{i}^{\prime}}(L) \geq 2$, then there is a torus $T_{j}^{\prime}$ with $j \neq i$ and $T_{i}^{\prime}=T_{j}^{\prime}$, i.e. $V_{i}^{\prime} \cap V_{j}^{\prime}=T_{i}^{\prime}$ and $V_{i}^{\prime} \cup V_{j}^{\prime}=S^{3}$.

Let $\mathcal{T}_{\mu}=\left\{T_{1}^{\prime}, \ldots, T_{\nu}^{\prime}\right\}$. The existence of tori in $\mathcal{T}_{\mu}$ depends on the uncertainty of the atoroidal decomposition of the Seifert pieces. So each $T_{i}^{\prime}$ is a connectable torus with discrepancy zero. By an $m_{i}$-twist $g_{i}^{\left(m_{i}\right)}$ along $V_{i}^{\prime}$, the discrepancy of $g_{i}^{\left(m_{i}\right)}\left(T_{i}^{\prime}\right)$ gets to be $m_{i}$. So $g_{i}^{\left(m_{i}\right)}$ produces no similar tori in $g_{i}^{\left(m_{i}\right)}\left(E^{\prime}\right)$ for any $m_{i} \neq 0$. 
Consider the general cases. Let $\Phi$ be any composition of sequential twists, each of which is defined after applying the previous twists. We classify these twists up to isotopy as follows: The first class consists of all the twists already defined, such as $f_{1}^{\left(n_{1}\right)}, \ldots, f_{m}^{\left(n_{m}\right)}$; The second class consists of all the twists defined after applying the twists in the first class, such as $g_{c(1)}^{\left(m_{e(1)}\right)}, \ldots, g_{c\left(k_{1}\right)}^{\left(m_{c\left(k_{1}\right)}\right)}$ with $T_{c(1)}^{\prime}, \ldots, T_{c\left(k_{1}\right)}^{\prime}$ being mutually disjoint; Similarly, each class consists of the twists defined for the first time after applying the twists in the previous classes.

Calculate $\Phi$ using $f_{i}^{\left(n_{1}\right)} \circ f_{i}^{\left(n_{2}\right)}=f_{i}^{\left(n_{1}+n_{2}\right)}$ in the first class and $g_{j}^{\left(m_{1}\right)} \circ g_{j}^{\left(m_{2}\right)}=g_{j}^{\left(m_{1}+m_{2}\right)}$ in the second class. Ignore all 0-twists, since they are isotopic to the identity. Then by the above observation, there is no twists out of the first two classes. Therefore any cases can be reduced to Theorem 2.2 .

Proof of Theorem 2.2. Assume $f_{a(1)}^{\left(n_{1}\right)} \circ \cdots \circ f_{a\left(k_{1}\right)}^{\left(n_{k_{1}}\right)}(L) \cong f_{b(1)}^{\left(m_{1}\right)}{ }_{0} \cdots \circ$ $f_{b\left(k_{2}\right)}^{\left(m_{k_{2}}\right)}(L)$ for some $\left(n_{1}, \ldots, n_{k_{1}}, m_{1}, \ldots, m_{k_{2}}\right)$. Under this assumption, we show $\sum_{i=1}^{k_{1}} n_{i}-\sum_{j=1}^{k_{2}} m_{j}=0$ in the following.

There is an orientation preserving homeomorphism $\varphi: S^{3} \rightarrow S^{3}$ carrying $L_{1}=f_{a(1)}^{\left(n_{1}\right)} \circ \cdots \circ f_{a\left(k_{1}\right)}^{\left(n_{k_{1}}\right)}(L)$ to $L_{2}=f_{b(1)}^{\left(m_{1}\right)} \circ \cdots \circ f_{b\left(k_{2}\right)}^{\left(n_{k_{2}}\right)}(L)$. Note that $\varphi \circ f_{a(1)}^{\left(n_{1}\right)} \circ \cdots \circ f_{a\left(k_{1}\right)}^{\left(n_{k_{1}}\right)}\left(U\left(\mathcal{T}-\mathcal{T}_{C}\right)\right)$ is isotopic to $f_{b(1)}^{\left(m_{1}\right)} \circ$ ..० $f_{b\left(k_{2}\right)}^{\left(m_{k_{2}}\right)}\left(\bigcup\left(\mathcal{T}-\mathcal{T}_{C}\right)\right)$. Modify $\varphi$ by an isotopy so as to satisfy $\varphi \circ$ $f_{a(1)}^{\left(n_{1}\right)} \circ \cdots \circ f_{a\left(k_{1}\right)}^{\left(n_{k_{1}}\right)}\left(\bigcup\left(T-\mathcal{T}_{C}\right)\right)=f_{b(1)}^{\left(m_{1}\right)} \circ \cdots \circ f_{b\left(k_{2}\right)}^{\left(m_{k_{2}}\right)}\left(\bigcup\left(\mathcal{T}-\mathcal{T}_{C}\right)\right)$. Then $\varphi$ carries each maximal twisted Seifert manifold to a maximal twisted Seifert manifold. So modify $\varphi$ so as to preserve the fibering of maximal twisted Seifert manifolds. Suppose $\mu_{i}=n_{a(k)}-m_{b\left(k^{\prime}\right)}$ when $i=a(k)=b\left(k^{\prime}\right)$ for some $k$ and $k^{\prime}, \mu_{i}=n_{a(k)}$ when $i=a(k)$ and $i \neq b\left(k^{\prime}\right)$ for any $k^{\prime}, \mu_{i}=-m_{b(k)}$ when $i=b(k)$ and $i \neq a\left(k^{\prime}\right)$ for any $k^{\prime}$, and otherwise $\mu_{i}=0$.

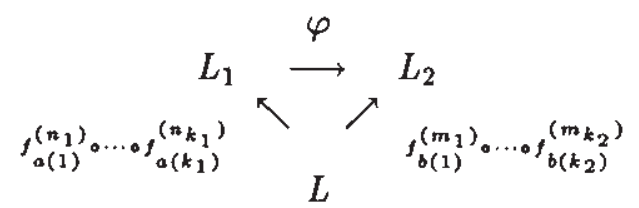


We classify the solid tori to twist in the two cases. First consider the case of the solid tori without connectable toral boundaries. Let $\psi: S^{3} \rightarrow S^{3}$ be a discontinuous map defined by

$$
\psi=\left(f_{b(1)}^{\left(m_{1}\right)} \circ \cdots \circ f_{b\left(k_{2}\right)}^{\left(m_{k_{2}}\right)}\right)^{-1} \circ \varphi \circ\left(f_{a(1)}^{\left(n_{1}\right)} \circ \cdots \circ f_{a\left(k_{1}\right)}^{\left(n_{k_{1}}\right)}\right)
$$

In this case, we can decompose the set $\mathcal{T}-\mathcal{T}_{C}$ to the orbits $\left\{T_{p(i, 1)}, \ldots, T_{p\left(i, N_{i}\right)}\right\}(1 \leq i \leq r)$ under $\psi$. When $T_{p(i, 1)}$ bounds a hyperbolic piece, in the same way as the proof of Theorem 2.1 , we get $\sum_{j=1}^{N_{i}} \mu_{p(i, j)}=0$ for any $i$ by Lemma 4.1. When $T_{p(i, 1)}$ bounds a twisted Seifert manifold $M, \psi^{N_{i}}$ gives a fiber preserving map of $M$ and an orientation preserving self-homeomorphism of $T_{p(i, 1)}$. Then we get $\sum_{j=1}^{N_{i}} \mu_{p(i, j)}=0$ by Lemma 5.1 .

Next consider the case of the solid tori with the connectable toral boundaries. Let $T_{i} \in T_{C}$. Since each connectable torus has unique discrepancy, the sum of the discrepancy of all the connectable tori in the exterior of $L_{1}$ and $L_{2}$ are the same. On the other hand, $f_{i}^{(n)}$ increases the discrepancy of $T_{i}$ by $n$. Therefore the sum of $n_{i}$ with $T_{a(i)} \in T_{C}$ is equal to that of $m_{j}$ with $T_{b(j)} \in \mathcal{T}_{C}$, hence $\sum_{T_{i} \in T_{C}} \mu_{j}=0$.

Consequently we get $\sum_{t_{i} \in T} \mu_{i}=0$, that is, $\sum_{i=1}^{k_{1}} n_{i}-\sum_{j=1}^{k_{2}} m_{j}=0$, and this completes the proof.

\section{PROOF OF THEOREM 2.3}

In this section, we suppose $V_{c(1)} \subset V_{c(2)} \subset \cdots \subset V_{c(k)}, \omega_{V_{c(1)}}(L) \geq$ 2 , and $\omega_{V_{c(i+1)}}\left(\left(L-V_{c(i)}\right) \cup \operatorname{coreV}_{c(i)}\right) \geq 2$ for $1 \leq i \leq k-1$. Assume $f_{c(1)}^{\left(n_{1}\right)} \circ \cdots \circ f_{c(k)}^{\left(n_{k}\right)}(L) \cong L$ for some $\left(n_{1}, \ldots, n_{k}\right)$. There is an orientation preserving homeomorphism $\varphi: S^{3} \rightarrow S^{3}$ carrying $f_{c(1)}^{\left(n_{1}\right)} \circ \cdots \circ f_{c(k)}^{\left(n_{k}\right)}(L)$ to $L$. Note that $\varphi \circ f_{c(1)}^{\left(n_{1}\right)} \circ \cdots \circ f_{c(k)}^{\left(n_{k}\right)}\left(\bigcup\left(\mathcal{T}-\mathcal{T}_{C}\right)\right)$ is isotopic to $\bigcup\left(T-\mathcal{T}_{C}\right)$ 
in E. Modify $\varphi$ so as to satisfy $\varphi \circ f_{c(1)}^{\left(n_{1}\right)} \circ \cdots \circ f_{c(k)}^{\left(n_{k}\right)}\left(U\left(T-T_{C}\right)\right)=$ $\bigcup\left(T-\mathcal{T}_{C}\right)$. Denote $\varphi \circ f_{c(1)}^{\left(n_{1}\right)} \circ \cdots \circ f_{\mathrm{c}(k)}^{\left(n_{k}\right)}$ by $\varphi_{\mu}$ where $\mu=\left(n_{1}, \ldots, n_{k}\right)$.

Lemma 6.1. $T_{c(i)} \in \mathcal{T}-\mathcal{T}_{C}$ implies $n_{i}=0$.

Proof. Assume $T_{c(i)} \in \mathcal{T}-\mathcal{T}_{C}$. Since $\varphi_{\mu}$ fixes $U\left(\mathcal{T}-\mathcal{T}_{C}\right)$, we can consider the orbit of $T_{c(i)}$ under $\varphi_{\mu}$. Either $V_{c(i)} \subset$ int $V_{c(j)}$ or $V_{c(j)} \subset$ int $V_{c(i)}$ holds for any $j \neq i$, therefore $T_{c(j)}$ does not belong to the orbit of $T_{c(i)}$ under $\varphi_{\mu}$. According as $M_{c(i)}$ is a hyperbolic piece or a Seifert piece, the proof completes by Lemma 3.2 or 5.1 in the same way as Lemma 4.1 respectively.

By Lemma 6.1, we can assume $T_{c(i)} \in \mathcal{T}_{C}$ for any $i$. Then each $T_{c(i)}$ is contained in a maximal twisted Seifert manifold.

Lemma 6.2. $n_{i}=0$ for any $i$. $i$

Proof. Ignoring $f_{c(i)}^{\left(n_{i}\right)}$ with $n_{i}=0$, we can suppose $n_{i} \neq 0$ for any

First assume $T_{c(i)} \in \mathcal{T}_{D} \cap \mathcal{T}_{C}$ for any $i$. Since $\varphi_{\mu}(L)=L$ implies that the torus decomposition of their exteriors give the same number of tori, then $\varphi_{\mu}\left(T_{c(i)}\right) \in \mathcal{T}_{D} \cap \mathcal{T}_{C}$ for any $i$. Therefore $\varphi_{\mu}\left(T_{c(i)}\right)$ is a connectable torus with discrepancy non-zero. In this case, $\varphi_{\mu}\left(\bigcup \mathcal{T}_{D}\right)$ is isotopic to $\bigcup \mathcal{T}_{D}$ in $E$. Then modify $\varphi$ so as to satisfy $\varphi_{\mu}\left(\bigcup \mathcal{T}_{D}\right)=\bigcup \mathcal{T}_{D}$. So we can consider the orbit of $T_{c(i)}$ under $\varphi_{\mu}$. Since $T_{c(1)}, \ldots, T_{c(k)}$ are ordered by the inclusion relation of $V_{c(1)}, \ldots, V_{c(k)}$, their orbits under $\varphi_{\mu}$ are mutually different. According as $M_{c(i)}$ is a hyperbolic piece or a Seifert piece, we get $n_{i}=0$ by Lemma 3.2 or 5.1 , contradiction.

Next assume $T_{c(i)} \in T_{C}-\mathcal{T}_{D}$ for some $i$. Then $T_{c(i)}$ is a connectable torus with discrepancy zero. Suppose $V_{c(i)}$ is innermost of all the solid tori $V_{c(t)}$ with $1 \leq t \leq k$ and $T_{c(t)} \in \mathcal{T}_{C}-\mathcal{T}_{D}$. We define some solid tori $W_{1}, \ldots, W_{t}$ inductively in the following. Let $W_{1}=V_{c(i)}$. Since the discrepancy of $\partial W_{1}=T_{c(i)}$ is zero, that of $\varphi_{\mu}\left(\partial W_{1}\right)$ is $n_{i} \neq 0$. Then $\varphi_{\mu}\left(\partial W_{1}\right)$ is isotopic to a torus in $\mathcal{T}_{D}$. So modify $\varphi_{\mu}$ by an isotopy in $E$ so as to satisfy $\varphi_{\mu}\left(\partial W_{1}\right) \in T_{D}$. Then one of the following occurs:

(1) $\varphi_{\mu}\left(W_{1}\right)$ is equal to one of $V_{c(1)}, \ldots, V_{c(k)}$. 
(2) $\varphi_{\mu}\left(\partial W_{1}\right)=T_{c(j)}$ and $\varphi_{\mu}\left(V_{c(i)}\right) \neq V_{c(j)}$ for some $j$.

(3) $\varphi_{\mu}\left(\partial W_{1}\right)$ is disjoint to any of $T_{c(1)}, \ldots, T_{c(k)}$.

When either (2) or (3) holds, let $W_{2}=\varphi_{\mu}\left(W_{1}\right)$. Then the discrepancy of $\partial W_{2}$ is $n_{i} \neq 0$. Similarly, when the solid torus $W_{t}$ is defined, one of the following occurs:

(1) $\varphi_{\mu}\left(W_{t}\right)$ is equal to one of $V_{c(1)}, \ldots, V_{c(k)}$.

(2) $\varphi_{\mu}\left(\partial W_{t}\right)=T_{c(j)}$ and $\varphi_{\mu}\left(W_{t}\right) \neq V_{c(j)}$ for some $j$.

(3) $\varphi_{\mu}\left(\partial W_{t}\right)$ is disjoint to any of $T_{c(1)}, \ldots, T_{c(k)}$.

When either (2) or (3) hods, let $W_{t+1}=\varphi_{\mu}\left(W_{t}\right)$. The discrepancy of $\partial W_{t}$ is $n_{i} \neq 0$, and so is that of $\partial W_{t+1}$. The isotopy classes of essential tori in $E$ are finite, then we get $W_{t}=V_{c(j)}$ for some $t$ and $j$. Here the discrepancy of $T_{c(i)}$ and $T_{c(j)}$ are zero and $n_{i} \neq 0$ respectively, therefore $j \neq i$ holds. Note that $T_{c(\nu)} \subset \operatorname{int}\left(V_{c(i)} \cap E\right)$ implies $T_{c(\nu)} \in T_{D}$ for any $\nu$. Then the number of Seifert pieces in $W_{t} \cap E$ is less than or equal to that in $V_{c(i)} \cap E$, and the number of the isotopy classes of essential tori in $W_{t} \cap E$ is greater than or equal to that in $V_{c(i)} \cap E$. Then $V_{c(i)} \subset$ int $W_{t}$ contradicts the former, and $W_{t} \subset$ int $V_{c(i)}$ contradicts the latter. This completes the proof.

Consequently, these lemmas completes the proof of Theorem 2.3.

\section{References}

[I1] Ikeda, T. Atoroidal decompositions of link exteriors. Kobe J. of Math. 9 (1992), p. 71-88.

[I2] Ikeda, T. Twisting of knots along knotted solid tori. Tokyo J. of Math. 16 (1993), p. 147-154

[Ja] Jaco, W.H. Lectures on three-manifold topology. Conference board of Math., Amer. Math. Soc., 1980.

[JS] Jaco, W.H. and Shalen, P. Seifert fibered spaces in 3-manifolds. Mem. Amer. Math. Soc. 220, 1979.

[Jo] Johannson, K. Homotopy equivalence of 3-manifolds with boundaries. Lecture Notes in Math. 761, Springer-Verlag, 1979. 
[KMS1] Kouno, M., Motegi, K. and Shibuya, T. Twisting and knot types. Preprint.

[KMS2] Kouno, M., Motegi, K. and Shibuya, T. Behavior of knots under twisting. To appear in Advanced Studies in Pure Math.

[T] Thurston, W. The geometry and topology of 3-manifolds. Lecture Note, Princeton Univ., 1978.

NEC Corporation

Recibido: 17 de marzo de 1993

Information Technology Research Laboratories

1-1, Miyazaki 4-Chome, Miyamae-Ku,

Kawasaki, Kanagawa

216 Japan 